\title{
Programación Curricular Inclusiva en la Escuela Secundaria de Córdoba (Argentina) para Estudiantes con Trastorno del Espectro Autista (TEA)
}

\author{
Inclusive Curriculum Programming in a Secondary School of Córdoba \\ (Argentina) for Students with Autism Spectrum Disorder (ASD)
}

María Laura Grosso ${ }^{1}$ (id

Recibido: 25-01-2021; Aceptado: 12-04-2021; Publicado: 28-04-2021.

\section{RESUMEN}

Para poder enseñar de un Modo Inclusivo, se requiere un marco legal que permita diseñar el Programa Curricular y que considere el estilo de aprendizaje atípico del Estudiante con diagnóstico. El Autismo es una condición con características específicas cuya consideración es necesaria a la hora de diseñar programas inclusivos en la escuela. Objetivo. Abordar la normativa que permite la Inclusión y la Programación Curricular Inclusiva para Estudiantes con diagnóstico de Trastorno del Espectro Autista (TEA) en la Escuela Secundaria argentina de la Provincia de Córdoba. Método. Este artículo reflexivo describe las dimensiones diagnóstico del Autismo para poder considerar una Inclusión según su estilo de aprendizaje. Luego, se analizan los paradigmas que han impulsado los marcos regulatorios en el ámbito sanitario y educativo para la Inclusión de Aprendices con Autismo, en el secundario. Se coteja la programación posible para el TEA, según dicha normativa. Conclusión. Se propondrán las configuraciones de apoyo para eliminar las barreras a la participación, promover el Aprendizaje Significativo y se planteará un diseño general que considere objetivos, metodologías, estrategias y evaluaciones de Inclusión en el Aula.

Palabras clave: Legislación inclusiva; normativa Argentina; normativa de Córdoba; normativa sanitaria; normativa educativa; didáctica inclusiva; Estudiantes con TEA; Escuela Secundaria.

\section{ABSTRACT}

In order to teach in an Inclusive Mode, a legal framework is required that allows the Curriculum Program to be designed and that considers the atypical learning style of the Student with a diagnosis. Autism is a condition with specific characteristics whose consideration is necessary when designing inclusive programs at school. Objective. Address the regulations that allow Inclusion and Inclusive Curriculum Programming for Students diagnosed with Autism Spectrum Disorder (ASD) in the Argentine Secondary School of the Province of Córdoba. Method. 
This reflective article describes the diagnostic dimensions of Autism in order to consider an Inclusion according to its learning style. Then, the paradigms that have promoted the regulatory frameworks in the health and educational field foro the Inclusion of Learners with Autism, in secondary school, are analyzed. The possible programming for the TEA is checked, according to said regulations. Conclusion. Support configurations will be proposed to eliminate barriers to participation, promote Meaningful Learning and a general design will be proposed that considers objectives, methodologies, strategies and evaluations of Inclusion in the Classroom.

Keywords: Inclusive legislation; Argentine regulations; Córdoba regulations; health regulations; educational regulations; inclusive didactics; Students with ASD; secondary school.

\section{INTRODUCCIÓN}

Las personas con Trastorno del Espectro Autista (TEA) presentan características propias que, según la Organización Mundial de la Salud (OMS) y la American Psychological Association (APA), tiene que ver con dificultades en el área de la lingüística y la comunicación, patrones restringidos y repetitivos de comportamiento (OMS, 1990, 2018; APA, 2013). Las personas con dicho diagnóstico pueden entrar al sistema educativo desde que existe la ley que reconoce dicha identidad diagnóstica (TEA) y certifica su Inclusión; por lo que se requieren dos marcos legislativos: uno de salud y otro educativo. Estas dos instituciones deben convergir en la categoría del "Estudiante con un diagnóstico de TEA" para el ejercicio de sus derechos: implementar el proceso de enseñanza-aprendizaje en función de dicha certificación.

Ahora bien, ¿cómo se lleva adelante la Inclusión en Córdoba y cuáles son los Lineamientos Curriculares a los que se acogen los Estudiantes con Autismo en la Escuela Secundaria? Aquí, se abordarán los paradigmas que han impulsado los marcos regulatorios en el ámbito sanitario y educativo para la Inclusión de dichos Aprendices, en la provincia de Córdoba, Argentina. El análisis permite dar cuenta de los paradigmas que los sostienen describiendo los logros en materia inclusiva y reflexionando sobre los aspectos a mejorar.

Asimismo, con relación a la programación para Estudiantes con TEA, se propondrán los aspectos inclusivos que considerarán las configuraciones de apoyo para eliminar las barreras a la participación, promover el aprendizaje y plantear objetivos, metodologías, estrategias y evaluaciones de Inclusión en el aula.

La conclusión señala los aspectos legales a modificar y propone las dimensiones a considerar en una enseñanza inclusiva según el estilo de aprendizaje del Estudiante con Autismo. Otra consecuencia didáctica es una guía general para una propuesta de diseño y construcción del programa curricular para Estudiantes con TEA que se puede adaptar a cada materia, ya que hace referencia a los aspectos a potenciar o a minimizar según las características diagnósticas del Autismo.

\section{EL TRASTORNO DEL ESPECTRO AUTISTA (TEA)}

El Trastorno del Espectro Autista es una condición que ha pasado por diferentes clasificaciones. Desde la creación del vocablo autista (Bleuler, 1911) hasta hoy, las descripciones del comportamiento de los TEA han pasado por diferentes categorías nosográficas y por muchas discusiones en el ámbito de la salud mental (Grosso, 2021b). Actualmente, el Autismo aparece clasificado como un trastorno del neurodesarrollo tanto en el nuevo Diagnostic and Statistical Manual of Mental Disorders (DSM-5) de la APA (2013), como en el último manual de la OMS (2018), la Clasificación Internacional Estadística de Enfermedades y Problemas Relacionados con la Salud (CIE-11). 
EI TEA mantiene los síntomas clínicos identificados por Kanner (1943) y por Asperger (1944). En general, ambos dan cuenta de problemáticas persistentes en la comunicación, en la interacción social, patrones de comportamiento restrictivos y repetitivos, repertorio restringido de intereses y actividades, características estas que fueron consideradas una tríada para la construcción del cuadro sindrómico postulado por Wing y Gold (1979) y que sirvieron para los manuales de enfermedades: el DSM (APA, 2013, 2002, 1994) y la CIE (OMS, 2018, 1990).

Las explicaciones de los síntomas del TEA parten de perspectivas psicodinámicas, socioafectivas, cognitivas, sociocomputacionales, biológicas (Grosso, 2020a). Pero, ningún modelo canónico por sí solo los explica suficientemente y no todos son específicos del Autismo. Algunas dificultades en la interacción social, la comunicación y el lenguaje, son explicadas, en parte, por las teorías cognitivas de la mente (TM) (Baron-Cohen, Leslie y Frith, 1985), por la teoría sociocomputacional de control (Frawley, 2000), la teoría de la relación interpersonal del modelo afectivo (Hobson, 1990, 1991, 1993) y el sistema de las Neuronas Espejo (NS) (Rizzolatti y Craighero, 2004). Algunos patrones comportamentales restringidos, con un cotidiano estresante, lo explicarían las funciones ejecutivas (FE) (Ozonoff, Pennington y Rogers, 1991), el modelo afectivo y las NS; la falta de planificación, inhibición y control del comportamiento y las representaciones mentales, por las FE, el déficit de control, por la TM y las NS. La fortaleza en ciertas habilidades lo sostiene el modelo de la Coherencia Central (CC) (Frith y Happé, 1994; Frith, 2016).

Dichas explicaciones permiten comprender el continuum del TEA y, también, cuáles son los presupuestos humanos que, automática e implícitamente, posibilitan modos de interrelacionarse, comunicarse y comportarse. Las personas con Autismo tienen requerimientos específicos en la explicitación de la situación relacional y comunicativa, en el control externo, en ayudas organizativas, en programación secuencial, en la direccionalidad, en un mayor tiempo para procesar y en soportes como las TIC. La ausencia o exceso de estos requisitos es justamente parte de la descripción sintomatológica del Autismo.

Las implicaciones escolares son variadas (Grosso, 2020a). El aprendizaje no funciona igual para todos, porque se aprende de un modo diferente según los procesos que subyacen, el tipo de experiencias y el desarrollo neuropsicológico que se tuvo. El estilo de aprendizaje de las personas con TEA evidencia las necesidades educativas (NE) específicas que derivan de su condición diagnóstica (Grosso, 2020), por lo que la Escolarización Secundaria Inclusiva del alumnado con Autismo plantea complejos desafíos relacionados fundamentalmente con las dimensiones diagnósticas que requieren configuraciones de apoyo y adecuaciones curriculares.

\section{LA NORMATIVA ARGENTINA}

La Ley de Educación Común 1420 argentina de 1884, estableció la educación primaria, obligatoria y gratuita. A nivel nacional, la Ley 26.061 de Protección Integral de los Derechos de Niños, Niñas y Adolescentes (2005), sustentada en el principio de Interés Superior del Niño (la Convención sobre los Derechos del Niño (CIDN, UNESCO, 1989) fue ratificada en 1990 y adquirió rango constitucional en 1994. Esta estableció pautas federales y regulaciones para el mejoramiento de la enseñanza, el aprendizaje y las trayectorias escolares, en el nivel inicial, primario y modalidades. La Resolución 134 del Consejo Federal de Educación (CFE) aprobó estrategias para mejorar las trayectorias en los diferentes niveles, asegurando el derecho a la educación, favoreciendo el ingreso, tránsito y egreso de Estudiantes.

La Ley 26.206 de Educación Nacional de 2006 habla de educación especial para afrontar todas aquellas problemáticas específicas que no puedan ser abordadas por la educación común. El Ministerio de Educación, Ciencia y Tecnología, en acuerdo con el CFE, garantiza la Inclusión "de los Estudiantes con discapacidades en todos los niveles y modalidades según las posibilidades de cada persona" (Ley 26.206, 2006, Art. 42). Ese mismo año, había sido adoptada la CIDPD 
(UNESCO, 2006), aprobada en 2008, y adquirió jerarquía constitucional en el 2014 con la Ley No 27.044. La CIDPD adopta el modelo de la International Classification of Functioning, Disability and Health (ICF, en español CIF) (OMS, 2001) que considera a la persona en su totalidad, desde una perspectiva bio-psico-social. Basándose en un perfil de funcionamiento y en un análisis del contexto, la CIF permite individuar las barreras del Estudiante, evitando la tipificación.

La Resolución CFE 154 (2011) garantiza el ordenamiento y la cohesión del Sistema Educativo, en lo que respecta a la organización y articulación de los niveles. Igualmente, la Resolución CFE 155 de educación especial explicita la definición de orientaciones para la modalidad de Inclusión de las personas con discapacidad con derechos universales en todo el sistema nacional. La Resolución CFE 174 (2012) garantiza la trayectoria y permanencia en el nivel inicial de los Estudiantes. Los aprendizajes son indicadores de acreditación que permiten el pase de nivel.

En 2014, se promulga la Ley 27.043 TEA cuyos contenidos aparecen en normativas internacionales y nacionales relativos a la protección de la Infancia y la Salud Mental. Es a partir de entonces que se puede formalizar en un proceso la Inclusión de Estudiantes con dicho diagnóstico en las instituciones educativas, con diseños curriculares adecuados a sus necesidades. La reglamentación de la ley de abordaje integral e interdisciplinario de las personas con TEA se hizo efectiva a través de un decreto del presidente argentino (777, 2019). Una de las consecuencias es una tardía coordinación con las autoridades sanitarias y educativas de las provincias sobre la planificación de formación del recurso humano, abordaje interdisciplinario e integral y las acciones necesarias a los fines de la completa Inclusión, en los diferentes niveles educativos, laborales y sociales, de acuerdo con lo establecido por la CIDPD. Asimismo, muchos artículos e incisos no se pueden reglamentar por decreto y deben hacerse por resoluciones, como son la articulación con los ministerios de salud y educación para difusión y campañas sobre concientización sobre el TEA (Art. 2, Inciso b) o con el Ministerio de Salud Pública (Art. 2, Inciso e, sobre las prestaciones necesarias para el abordaje del TEA; Art. 2, Inciso f-g, estudios epidemiológicos y estadísticos; Art. 2, Inciso h-i, implementación, en las diferentes jurisdicciones, de atención médica y protocolos de salud y sobre la detección del TEA; Art. 5, sobre los gastos que afronta la administración pública). Por lo que, se relegan derechos y obligaciones al sector familiar-privado (salud, educación, etc.).

Hasta que se haga efectiva la reglamentación de la ley TEA, está la Ley 22.431/24.901: la gestión del Certificado Único de Discapacidad (CUD), el documento que puede acreditar su condición y que pueden presentar las personas con TEA para entrar a las escuelas. El CUD es público, intransferible y de validez nacional. A través del CUD se accede a los derechos de las personas con discapacidad: los sistemas de salud, educativo, de transporte, etc.; el módulo de apoyo a la integración escolar y tratamientos en obras sociales u organismos estatales.

El módulo de apoyo a la integración escolar lo realiza el sistema de seguros médicos de la provincia, dejando en manos de profesionales de la salud la realización del perfil psicopedagógico que deriva de la certificación de discapacidad.

La elaboración del CUD sigue la normativa del Servicio Nacional de Rehabilitación (SNR, 2015) que utiliza dos clasificaciones de la OMS para diagnóstico y certificación. La primera es la CIE-10 (2001), con códigos que permiten la descripción de la/s condición/es de salud, según un paradigma clínico (el Autismo viene clasificado con el código F84 dentro de los Trastornos Generalizados del Desarrollo, TGD). La segunda, la CIF, brinda el marco conceptual para confeccionar un perfil de funcionamiento a través de cuatro componentes: funciones y estructuras corporales, actividad y participación, factores ambientales y factores personales, seleccionando, en cada uno, las categorías acordes para describir la situación de las personas. En la CIF, el Autismo está codificado en torno a funciones psicosociales globales (b.121): "funciones mentales generales y su desarrollo a lo largo del ciclo vital, requeridas para entender e integrar de forma constructiva varias funciones mentales que conducen a la obtención de habilidades interpersonales necesarias para establecer interacciones sociales recíprocas tanto a lo referente al significado y a la finalidad" (CIF, 2001, p. 52). 
La certificación por discapacidad sigue los lineamientos del CIE-10; ahora bien, la normativa es ecléctica y hasta contradictoria en la forma de diagnosticar y evaluar el TEA. Por un lado, dice que se utilizan dos clasificaciones diagnósticas de la OMS, sin embargo, la evaluación sigue los parámetros del manual de la APA, el DSM-5 (SNR, 2015, p. 5). El DSM-5 habla del TEA, no del TGD. En el manual de la APA, no hay distinción entre Autismo infantil y adulto, el TEA no es triádico en el DSM-5 ni es considerado un trastorno del neurodesarrollo y la sigla es diferente (TEA-TGD). Por lo tanto, ambos manuales poseen paradigmas diferentes que, además de no ser compatibles, pueden generar problemáticas derivadas de diagnósticos diversos. La diferente clasificación no solo se presta a confusión terminológica, sino que una diagnosis mal escrita puede derivar en la no aplicación de la ley de las personas con discapacidad perdiendo los derechos que de ella se derivan, como la educación inclusiva; o la aplicación de protocolos y diseños curriculares que no se adecuan al perfil pedagógico del Estudiante con Autismo.

Otra de las contradicciones que se presenta entre la ley TEA y el paradigma de salud mental del CIE-10, es la de presentar un enfoque centrado en la enfermedad. Su posible aplicación al campo de la infancia y adolescencia no considera el principio de interés superior del niño, en tanto reconocimiento de su subjetividad. El centrarse en la enfermedad supone partir de una situación de déficit que se contradice con los principios contenidos en la CIDPD y la OMS basados en la capacidad funcional en un ambiente favorecedor o perjudicial del desarrollo personal.

Las políticas enmarcadas en una concepción universal del derecho han de promover la Inclusión, contemplando las necesidades de las personas, integrando las diferencias subjetivas en un ambiente favorecedor (Grosso, 2020c), pero no la exclusión a derechos fundamentales. Las leyes por patologías funcionan estigmatizando, discriminando o marginando y favorecen la lógica mercantil. Al no haber obligación estatal de tutelar la cobertura universal, se restringe el acceso a prestaciones efectivas y las acciones repercuten en manos de los particulares, o sea, la familia.

A nivel nacional, existe la Resolución Ministerial 311 (CFE, 2016) que regula los procesos de Inclusión escolar, certificación y titulación de los trayectos de Estudiantes que presentan necesidades educativas (NE) derivadas de la discapacidad donde se incluye al TEA; propicia y garantiza las condiciones para la Inclusión al interior del sistema educativo para el acompañamiento de las trayectorias de Estudiantes con discapacidad. Igualmente, establece que las escuelas tienen prohibido rechazar la inscripción o reinscripción de un Estudiante por motivos de discapacidad (discriminación). Asimismo, predispone un Proyecto Pedagógico Individual (PPI) para Estudiantes con diagnóstico de discapacidad en los Niveles Primario y Secundario. Enuncia que los mismos deben ser evaluados y calificados según ese PPI. Esas evaluaciones y calificaciones determinan el pase de año o de nivel.

El Estudiante con TEA entra con diagnóstico que indica: nivel de Autismo, intereses, comportamientos $\mathrm{y} / \mathrm{o}$ actividades persistentes desarrolladas, describe la predisposición del Estudiante con pares, con el Docente, con contenidos curriculares para configurar estrategias para favorecer la comunicación e interacción social.

\section{La normativa de la Provincia de Córdoba}

El sistema federal argentino garantiza la autonomía provincial. Muchas leyes nacionales sancionadas para toda la república se reglamentan y cumplen una vez que las provincias las replican. Córdoba no regula los procesos para la aplicación de la ley TEA, pero la incluye a través de otra normativa.

La Resolución Provincial Ministerial 1114 (2000) certifica y acredita el paso por la institución escolar de los Estudiantes con NE Especiales (NEE) con sus respectivas adaptaciones curriculares. Estas las realizan los Docentes de la institución, acompañadas por la firma de los responsables 
y directivos. La Resolución Ministerial 635 (2008) amplía la 1114 y dispone su adecuación para optimizar los procesos de integración escolar de Estudiantes con NEE en el Secundario, a los que se les hayan implementado adecuaciones curriculares significativas o un programa de diversificación curricular. La Resolución dispone que a toda certificación de estudios se acompañe una leyenda y un Informe de las Competencias Adquiridas elaborados por Docentes. A cada uno le corresponde la realización de la propia disciplina.

La Resolución Ministerial 667 (2011) regula los procesos de integración en cuanto a: 1) diagnóstico de orientación escolar 2) roles, funciones y responsabilidades del Docente de Apoyo, en particular de equipos privados, 3) compromisos de los diferentes actores implicados en estos procesos y 4 ) rol del equipo directivo.

De todos modos, actualmente, a nivel provincial, existe la Resolución Ministerial 311 (2016) que amplía la Resolución Ministerial 667/11, con el fin de regular los procesos de integración escolar, certificación y titulación de los trayectos de Estudiantes que presentan NEE en el Nivel de Educación Secundaria en la que se contempla al Estudiante con TEA, siempre y cuando entre con un CUD.

El Memorándum 02 (2017) corrige la denominación de NEE a Necesidades Educativas Derivadas de Discapacidad (NEDD) y amplía la posibilidad de ingreso al sistema educativo de personas con TEA sin presentar CUD, sin obligatoriedad.

Los Lineamientos de la Política Educativa de la Provincia de Córdoba 2016-2019 establecen la "Elaboración de un protocolo con orientaciones pedagógicas-didácticas destinadas a los equipos directivos y Docentes del Nivel, referidas a la integración de niños con necesidades educativas derivadas de la discapacidad" (Ministerio de Educación, 2016, p. 4). Las NE, de donde derivan las intervenciones escolares para la Inclusión, se certifican en base a un diagnóstico médico de discapacidad que, como se vio, resulta ecléctico, poco preciso y claro.

Córdoba aún no adhiere a la Ley Nacional TEA (Ley 27.043, 2014), por lo tanto, su reglamentación no se llega a implementar, relegando derechos y obligaciones al sector familiar-privado (salud, educación, trámites), como a nivel nacional. Una de las consecuencias es la imposibilidad de coordinar con las autoridades sanitarias y educativas la planificación de la formación del recurso humano, el abordaje integral e interdisciplinario y las acciones necesarias a los fines de la completa Inclusión educativa, laboral y social, de acuerdo con lo establecido por la CIDPD. Por Ley 22.431, 24.901, está contemplada solo la gestión del CUD, para entrar al sistema educativo.

Al no haber obligación estatal de tutelar la cobertura universal, se restringe el acceso a prestaciones efectivas y las acciones repercuten en manos de los particulares. Una de ellas recae en el Docente de Apoyo a la integración, este Docente debería ser un maestro de Enseñanza Especial (código 13-455, resolución 667 (2011) del primario. Sin embargo, en la mayoría de los casos, el acompañante o Docente de Apoyo a la integración es un personal ajeno al Ministerio de Educación Pública, pertenece al sistema sanitario del ámbito privado, con costos, trámites y responsabilidades a cargo de la familia (aunque haya reintegro estatal), pueden ser psicólogos, psicopedagogos, psicomotricistas y otros equivalentes o supletorios. Por lo tanto, la formación del Docente de Apoyo a la integración pertenece mayoritariamente al ámbito médico, no al de la educación especial o integradora.

En síntesis, desde el Ministerio de Salud, el TEA está reconocido, aunque no reglamentado. Hay ambivalencias en cuanto a su entidad nosográfica, lo cual hace que, en la práctica, los diagnósticos se realicen con clasificaciones (DSM-5, CIE-10) imprecisas, resultando así una suma variada de siglas no compatibles (TGD, TEA, F84). La discapacidad está definida desde el paradigma clínico con contradicciones tanto en el reconocimiento de la categoría sindrómica, como en el reconocimiento de los derechos. Las prestaciones que se derivan de dicho diagnóstico 
no están tuteladas por el estado, relegando dicha condición al ámbito familiar o privado. Este no reconocimiento, no respeta el principio de las convenciones de Derechos Humanos (DDHH) acatado por Argentina (Ley 26.378, Ley 25.280), discriminando las atenciones, excluyendo servicios y derechos derivados del TEA.

\section{El Proyecto Pedagógico Individual (PPI)}

EI PPI para la Inclusión es el documento del aprendiz con TEA que contiene su información personal y su articulación con la escuela. Expresa las adecuaciones de acceso en el que se predisponen los espacios, los tiempos y el material necesarios para poder implementar el currículo:

1. Datos del Estudiante (incluído el Certificado de Discapacidad, CUD),

2. Datos del padre y/o de la madre/ del tutor o encargado legal,

3. Datos de escuela/s, del Nivel Escolar y de la Modalidad de Educación,

4. Datos de la escuela de origen y de la Modalidad de Educación,

5. Datos del equipo externo al Sistema educativo,

6. Denominación de los integrantes del equipo escolar interdisciplinario,

7. Barreras físicas, académicas y comunicacionales,

8. Recursos tecnológicos, materiales y apoyos en formatos accesibles,

9. Participación familiar en el proceso de Inclusión.

También las adecuaciones específicas de la propuesta curricular concreta:

1. Propósitos, contenidos curriculares del Nivel escolar, con evaluación,

2. Incorporación de contenidos prioritarios de años anteriores,

3. Especificar contenidos no presentes en el Diseño del Nivel: autonomía, autodeterminación, movilidad, auto-cuidado, sociales, actividades diarias,

4. Acuerdos con el Docente de Apoyo para actividades y trabajo conjunto,

5. Configuraciones de apoyo implementadas,

6. Apoyos y Ajustes Razonables en objetivos, contenidos y secuencias, metodología, criterios y procedimientos de evaluación,

7. Organización del trabajo: estilo de aprendizaje, dinámica del grupo, modos de participación, recursos, modalidad áulica (colaborativo, etc.),

8. Información sobre instancias de educación no formal en la comunidad.

El proceso de evaluación establece los criterios, los instrumentos adecuados, la personalización del tiempo. Todo ello, se acuerda junto con el Docente de Apoyo. Para la promoción, se indica el grado/ciclo/año y se protocoliza con firmas.

Las barreras y obstáculos para la Inclusión escolar de Estudiantes con NEDD pueden ser de diferente tipo: barreras de acceso físico, de comunicación, didácticas o sociales/actitudinales (de los Docentes, de Estudiantes, de familiares, carencias de información, capacitación, conocimiento de los procesos inclusivos).

Configuraciones de apoyo son aquellas actividades que aumentan la capacidad de la escuela para atender a la diversidad. Para los Aprendices con TEA, estas son una respuesta a la necesidad de eliminar/minimizar las barreras y potenciar las habilidades. Para ello, se diseñan atenciones: con asesoramiento y orientación, capacitación, provisión de recursos, cooperación y acción coordinada de los miembros implicados (sean o no Docentes), seguimiento e investigación.

Por lo que, desde un punto de vista inclusivo, se expresa la consideración del aprendiz con diagnóstico certificado de TEA en la escuela según su condición y las limitaciones que puede 
encontrar. Esa expresión es la condición de posibilidad que permite viabilizar estrategias y metodologías educativas y mecanismos de formación del personal involucrado en el quehacer Docente. Sin embargo, no se explicitan las modalidades de abordaje a las barreras y limitaciones a la participación y aprendizaje de las NEDD ni de las configuraciones de apoyo.

\section{SÍNTESIS DE LOS MARCOS LEGALES}

En este apartado se sintetizan los paradigmas que articulan los documentos que predisponen la Inclusión de Estudiantes con TEA. EI PPI diseña las configuraciones de apoyo que se enuncian en las adaptaciones curriculares, para hacer accesible la transposición didáctica según los estilos de aprendizajes.

El estilo de aprendizaje del Estudiante con TEA viene establecido desde el ámbito de la salud pública en el PPI, expresado en el módulo de apoyo a la integración. La autoría es de una institución ajena a la escuela, aunque con acuerdos explícitos, que diseña el perfil pedagógico: la salud.

El PPI contiene información civil y las adecuaciones escolares y curriculares, y lo realizan los Docentes curriculares junto con el Docente de Apoyo a la Inclusión, la presentación recae en manos del mismo Docente de Apoyo.

El trabajo áulico del aprendiz con TEA en cada materia (lengua, matemáticas, etc.) se da en sinergia entre el Docente curricular y el de apoyo. Cada Docente expresa los acuerdos para la Inclusión junto con el Docente de Apoyo articulando los mandatos curriculares en función del estilo de aprendizaje.

En Córdoba, el CUD y el perfil del estilo de aprendizaje del Estudiante con TEA son dos ámbitos diferentes (salud y educación) que se trata en el ámbito de la salud con las contradicciones que pueden derivar de los diferentes paradigmas (sanitario o educativo) y las posiciones personales de los profesionales.

\section{DIDÁCTICA INCLUSIVA EN EL AULA}

La intervención didáctica hace referencia a las iniciativas e innovaciones que involucran a la planificación y la organización curricular, llegando a materializarse con la práctica de la programación. Cada Estudiante con Autismo tiene derecho a una programación individualizada, adaptada, ajustada, abierta, flexible y alternativa al plan de estudios, que contiene objetivos específicos y personales: el PPI.

La transposición inclusiva del objeto curricular en función del estilo de aprendizaje del Estudiante con TEA es competencia del Docente titular de materia, es decir, que le corresponden las decisiones motivadas de cada actividad y propuesta de intervención didáctica inclusiva específica.

El contexto de aprendizaje más efectivo para Aprendices con este diagnóstico es aquel desarrollado con un grado importante de organización, tanto mayor cuanto menor es la edad o el nivel de desarrollo. La intervención didáctica inclusiva ha de recorrer el proceso de andamiaje que va desde un grado alto de organización-estructuración a la desestructuración programada, paulatina y más cercana a los entornos culturales.

El objetivo de cada intervención didáctica es el Aprendizaje Significativo de cada materia (Ausubel, 1976). Para Estudiantes con TEA, las NE que puedan emerger según las dimensiones diagnósticas requieren de configuraciones de apoyo para potenciar las dificultades del conocimiento social, 
las habilidades comunicativas y lograr una conducta autorregulada, que son las dimensiones diagnósticas (CIE-10, 1990; DSM-V, 2013) sensibles a calibrar en el proceso educativo. Los objetivos de la intervención didáctica inclusiva son:

1. Incluir, favoreciendo el principio de educación en la diversidad

2. Organizar el contexto inclusivo para Aprendices con Autismo

3. Organizar un marco pedagógico, metodológico y curricular inclusivos

4. Trabajar en sinergia junto con Docente de Apoyo para mediar

5. Organización de recursos personales y materiales según las necesidades en los diferentes momentos educativos (aulas, recreos, etc.)

6. Conseguir la inserción del aula de apoyo en la dinámica general

7. Proponer configuraciones de apoyo para atender las NE del aprendiz

8. Dar respuesta a las NE de los Aprendices mediante el diseño de adaptaciones curriculares individualizadas

9. Desarrollar iniciativas inclusivas de colaboración e investigación con el profesorado, promoviendo la formación interna,

10. Coordinar con las familias el PPI para el logro de objetivos comunes.

Considerando el aprendizaje como un proceso y partiendo del perfil dinámico funcional del aprendiz con Autismo, las tareas a realizar conjugan los estilos de aprendizaje con los contenidos, habilidades y competencias. De acuerdo con ellos, se pueden aplicar técnicas metodológicas diversificadas y adaptadas a los estilos de aprendizajes de los Estudiantes con TEA. Al hablar de metodologías inclusivas, se hace referencia a los caminos seleccionados y orientados a la enseñanza que considera la diversidad.

El Docente de Apoyo es el instrumento en el proceso de aprendizaje que posibilita la mediación, las actividades individuales se realizan junto con él, aunque hay tareas que pueden involucrar a otros Estudiantes con funciones de tutor de otros compañeros para crear mayores propensiones a establecer relaciones educativas entre ellos. La aplicación de tutorías estimula a los tutores a recurrir a estrategias didácticas para enseñar a sus compañeros. Otra estrategia metodológica es el aprendizaje cooperativo donde trabajan, en grupos pequeños, juntos, Estudiantes con diferentes condiciones, para resolver un problema o completar un proyecto. El trabajo áulico se selecciona considerando las características del grupo.

La metodología didáctica requiere una doble labor: enseñar el contenido, la habilidad, la competencia, pero, también, explicitar su uso: adecuado, funcional, espontáneo y generalizado. Las configuraciones de apoyo representan un puente entre las NE y las barreras al aprendizaje y la participación, pero, entran en el proceso de enseñanza de un modo gradual y calibrado. Con el tiempo, el propósito es ir minimizando de un modo progresivo las ayudas.

La disposición de tareas inclusivas para el aprendizaje ha de proponer diferentes actividades, algunas sin error o evitando demasiadas variables que induzcan a error (multiple choice, seleccionar alternativas, opciones del parámetro, etc.), el proceso serial, la resolución de problemas con ayudas otorgadas, la identificación de la meta, la respuesta a preguntas etc., si finaliza con éxito las tareas disminuye la sensación de fracaso que genera ansiedad, desmotivación, confusión y hasta bloqueo. A continuación, la metodología de un aula inclusiva con un aprendiz con Autismo:

1. Seleccionar actividades diversificadas,

2. Priorizar métodos que favorezcan la integración e interacción a través de la experiencia directa, con situaciones reales y problemáticas concretas,

3. Usar estrategias de aprendizaje horizontal en el aula,

4. Planificar actividades que aborden los mismos contenidos a través de modalidades o lenguajes diferentes, que atiendan los estilos de aprendizaje, 
5. Favorecer estrategias que potencien la motivación y amplíen intereses,

6. Recurrir a estrategias lúdicas que unan contenidos con participación,

7. Diseñar actividades con diferentes grados de dificultad y niveles,

8. Pautar momentos para actividades diferentes, a elección,

9. Equilibrar las actividades en diferentes agrupamientos,

10. Ritualizar los diferentes momentos didácticos: inicio, desarrollo, cierre,

11. Anticipar la tarea a realizar, respetando acuerdos,

12. Explicitar cada paso de la tarea (qué cómo, etc.) de un modo simple,

13. Repetir y redundar en la explicitación de la tarea a través de modalidades o lenguajes diferentes, de modo concreto, breve y simple,

14. Ante imprevistos, naturalizar el cambio como estrategia de vida,

15. Calibrar soportes, recursos y ayudas para generar autonomía y/o según la tarea,

16. Usar evaluaciones variadas que ponderen los estilos de aprendizajes,

17. Acordar andamiaje del Docente de Apoyo como mediación específica.

Las estrategias didácticas son las decisiones orientadas, conscientes e intencionales que responden al cómo enseñar, tanto las estrategias como las metodologías se derivan del qué y a quién enseñar. Sin embargo, es posible delimitarlas dentro del marco inclusivo como las elecciones coordinadas, ordenadas y organizadas para inculcar, conectar y recuperar los conocimientos, las habilidades y competencias necesarios para cumplimentar una tarea, en función de la diversidad y de las condiciones específicas de la situación educativa. La función de las estrategias es seleccionar el camino más efectivo, productivo y creativo de enseñanza-aprendizaje. Bajo estos criterios, la Inclusión contempla estas estrategias:

1. Personalización graduada de enseñanza: andamiaje y mediación,

2. Regulación y autorregulación del aprendizaje,

3. Actividades de aprendizaje horizontal (tutorías, cooperativo, etc.),

4. Seccionar etapas encadenadas de las tareas,

5. Actividades cortas y variadas,

6. Alternancia pautada de actividades, recursos y apoyos,

7. El juego para integrar aprendizajes y promover la participación,

8. Promover la participación articulando estímulos variados,

9. Usar tecnologías y sistemas alternativos (auditiva-visual cinética),

10. Indicación y desvanecimiento de la ayuda con reducción paulatina,

11. Modelar el aprendizaje guiando la forma de realización de tarea,

12. Repetir, con diferente modalidad y tarea, la información, el tema/argumento/problema, los rituales (rutina),

13. Proporcionar indicaciones en diversas modalidades y lenguajes,

14. Contribuir al desarrollo de la autonomía de aprender a aprender,

15. Establecer un clima de participación y de cooperación,

16. Evaluar el proceso como instancia de acreditación,

La evaluación sigue el recorrido educativo realizado, articulando las tareas desarrolladas a los objetivos específicos programados en el PPI en función de métodos y estrategias utilizadas según el estilo de aprendizaje del Estudiante con Autismo y su perfil dinámico funcional. Ante la diversidad educativa, la evaluación responde con flexibilidad, apertura, diferenciación, una instancia que deriva del mismo proceso.

La evaluación es un momento del recorrido educativo que da cuenta tanto de la labor Docente como del trabajo del aprendiz. La evaluación responde a los objetivos y puede reformularse para ir ajustando la práctica a los aprendizajes. 
Asimismo, la evaluación va acordada anticipadamente con el aprendiz con TEA y el Docente de Apoyo, estableciendo criterios, instrumentos adecuados, tiempos y espacios. Además de la planificación y preparación previa, es aconsejable explicitar el tipo de formato a evaluar, según las tareas realizadas. Si las actividades respondieron a un aprendizaje horizontal, se evalúa el trabajo realizado por el grupo o pares ponderando la participación como un criterio más, considerando las barreras del aprendiz con TEA. Los portafolios, autoevaluaciones, evaluaciones entre compañeros, evaluaciones de proyectos, por ejemplo, son instancias que permiten examinar, además de los contenidos, habilidades y competencias, las dimensiones sensibles de participación, interacción, comunicación, autonomía y consciencia de sí en el trabajo realizado. La evaluación ha de ponderar el proceso realizado.

1. Personalización de la evaluación,

2. Criterios de evaluación según las adaptaciones del PPI,

3. Evaluar las decisiones metodológicas con el Docente de Apoyo,

4. Evaluar la autorregulación en dicha instancia ansiógena,

5. Evaluación de aprendizaje horizontal ponderada,

6. Seccionar y recuperar las tareas desarrolladas,

7. Evaluación breve, corta,

8. Calibrar las configuraciones de apoyo, con reducción de ayuda,

9. Uso de tecnologías y sistemas alternativos de comunicación aprendidos,

10. Proporcionar indicaciones en diversas modalidades y lenguajes,

11. Indicaciones concretas, claras y breves sin lenguaje figurado,

12. Hacer preguntas cerradas, sin alternativas ni posibilidades de error,

13. Usar un canal no combinado: oral o escrito,

14. Usar una modalidad perceptual o cognitiva no combinada,

15. Priorizar el canal y/o modalidad preferencial,

16. Priorizar la comprensión a la producción,

17. Contribuir al desarrollo de la autonomía con autoevaluaciones,

18. Evaluar el proceso considerando las barreras individuales,

19. Priorizar la evaluación formativa.

\section{CONCLUSIÓN}

Las personas con Autismo entran a la escuela con una identidad certificada establecida y regulada desde dos marcos legislativos: salud y educación. Estas dos instituciones convergen en la consideración de la existencia de la categoría Estudiante con una condición diagnosticada de TEA para el ejercicio pleno de sus derechos: el proceso de enseñanza-aprendizaje inclusivo de los mismos en función de su certificado. La escuela incluye si predispone una intervención didáctica individualizada y accesible en programas curriculares.

La Inclusión en Córdoba, Argentina, se realiza a través del derecho a la educación por tener discapacidad (Resolución Ministerial CFE, 311, 2016), pero poniendo en el centro la enfermedad (paradigma clínico), no desde el paradigma de DDHH. Dicha Inclusión especifica los lineamientos curriculares con los que se los acoge en la escuela, sin embargo, al no haber un marco legal en TEA específico, no se garantiza la formación, la investigación ni la reglamentación que vehiculice la procedimentalización en instituciones públicas, por ejemplo, se delega a los Docentes las adaptaciones de su materia, para luego integrarlos en el PPI: las competencias, conocimientos, estrategias y metodologías no están incluidas en la formación pedagógica (¿desde qué perfil pedagógico implementar una adaptación curricular sin formación en TEA?). Igualmente, los Docentes de apoyo, con formación en TEA, no pertenecen al ámbito educativo, por lo que no se garantiza su formación en educación. Asimismo, su sueldo depende del ámbito familiar o privado, no estatal. La Inclusión de personas con TEA, según la CIDPD, ha de expresarse en un marco que tutele sus derechos. 
La intervencióń individualizada en torno a las NE de cada aprendiz se ajusta a cada momento del desarrollo evolutivo atípico. Según lo previsto por las leyes, cada aprendiz con TEA tiene derecho a una programación individualizada, diversificada, adaptada, ajustada, abierta, flexible y alternativa al plan de estudios, que contiene una serie de objetivos específicos y personales. El PPI es el documento polifónico donde se expresan las voces de los Docentes para proponer la programación inclusiva, considerando a los padres o tutores y a las figuras profesionales específicas internas y externas a la institución escolar. Teniendo en cuenta el certificado de discapacidad y el estilo de aprendizaje del Estudiante, se establecen las adaptaciones curriculares con los objetivos, los recursos e instrumentos, las metodologías y las estrategias, las actividades didácticas y evaluaciones que coordinan las intervenciones educativas y se expresan en la programación.

Según los descriptores diagnósticos del TEA, se puede individuar, describir y explicar el estilo preferencial en que el Estudiante se comporta frente a la tarea de aprendizaje y se evidencian las NE que requieren configuraciones de apoyo. El estilo de aprendizaje del Estudiante con TEA da cuenta de requisitos específicos de preparación y anticipación de la tarea, con una atención guiada y calibrada de la información relacional relevante, de situaciones concretas, explícitas, con dosificada y calibrada carga cognitiva-emotiva interactiva, con espacios organizados y estructurados, permanentes y previsibles, con tiempos ralentizados, breves y flexibles, con preparación y anticipación al cambio, con guías personales, concretas, permanentes, referentes y sostenes emocionales, traductores de la cognición social.

El Docente de Apoyo a la integración es el adulto que pone el cuerpo (Grosso, 2020a) en el andamiaje (Bruner, 1986) del aprendizaje del Estudiante con TEA. Teniendo en cuenta que el aprendiz es una persona que además atraviesa el proceso de desarrollo psicofísico atípico (Vianello, 2011), el Docente de Apoyo favorece la construcción, creación, integración y el uso del conocimiento, las habilidades y competencias. Representa la herramienta dinámica (Vygotsky, 1964) que estructura, adapta, organiza, y guía el recorrido entre la zona de desarrollo real y la ZDP.

Pero, el Docente de Apoyo no diseña el contenido disciplinar de cada materia, sino el Docente de disciplina. Es decir, que le corresponden a este enseñante las decisiones motivadas de cada actividad y la propuesta didáctica. Los objetivos, estrategias, metodologías y evaluación de cada materia se articula con los documentos que presentan el perfil de destrezas y dificultades del aprendiz y las normativas curriculares. La intervención didáctica disciplinar involucra la planificación y la organización curricular y se materializa con la práctica de la programación.

El objetivo de la intervención didáctica para TEA es el aprendizaje de cada materia con las configuraciones de apoyo indicadas para las NE que puedan emerger. La potenciación del conocimiento social, de las habilidades comunicativas y sociales, como lograr una conducta autorregulada emergen como dimensiones sensibles a calibrar en el proceso educativo. Otros elementos a considerar en la programación para Aprendices con TEA tienen que ver ya con cada disciplina y son aspectos específicos de una didáctica inclusiva $n$ no ta general sino particular de cada materia.

\section{FINANCIACIÓN}

Este artículo deriva de la realización de una investigación para el Doctorado en Letras, de la UNC, Argentina.

\section{CONFLICTO DE INTERESES}

La autora declara que no tiene ningún conflicto de intereses. 


\section{REFERENCIAS}

American Psychological Association (1994). Diagnostic and statistical manual of mental disorders (4th). American Psychological Association.

American Psychological Association (2002). Manual diagnóstico y estadístico de los trastornos mentales. Texto revisado (DSM-IV-TR). Masson.

American Psychological Association-APA. (2013). Diagnostic and statistical manual of mental disorders (5th ed.). Arlington, VA. Recuperado de https://doi.org/10.1176/appi. books.9780890425596

Asperger, H. (1944). Die 'Autistischen Psychopathen' im Kindesalter. Archiv für Psychiatrie und Nervenkrankheiten, (117), pp. 76-136.

Ausubel, D. P. (1976). Psicología educativa. Un punto de vista cognoscitivo. México: Trillas.

Baron-Cohen, S., Leslie, A., \& Frith, U. (1985). Do children with autism have a theory of mind?. Cognition, 21, 37-46. https://doi.org/10.1016/0010-0277(85)90022-8

Bleuler, E. (1911). Dementia praecox or group of schizophrenia. In G. Aschaffenburg (Ed.), Handbook of Psychiatry. Special Section. 4th Division, 1st half (1-420). Leipzig and Vienna: Franz Deuticke.

Bruner, J. (1986). El habla del niño. Barcelona: Paidós.

Consejo Federal de Educación. (2011). Resolución 154 Pautas federales para el mejoramiento de la regulación de las trayectorias escolares en el nivel inicial, primario y modalidades. Buenos Aires, 31 de agosto de 2011. Recuperado de https://webcache.googleusercontent. com/search?q=cache: YVyxZb-FaEJ:https://cfe.educacion.gob.ar/resoluciones/res11/15411.pdf $+\& c d=2 \& h l=e s \& c t=c \mid n k \& g l=i t \& c l i e n t=$ safari

Consejo Federal de Educación. (2011). Resolución 155 de Educación Especial. Buenos Aires, 13 de octubre de 2011.

Consejo Federal de Educación. (2012). Resolución 174 Pautas Federales para el mejoramiento de la enseñanza y el aprendizaje y las trayectorias escolares en el nivel inicial, primario, modalidades, y su regulación. Buenos Aires, 13 de junio de 2012. Recuperado de http://abc. gob.ar/Secundaria/sites/default/files/res-cfe-174-12.pdf

Consejo Federal de Educación. (2016). Resolución 311 Promoción, acreditación, certificación y titulación de los Estudiantes con discapacidad. Buenos Aires, 15 de diciembre de 2016. Recuperado de https://www.argentina.gob.ar/sites/default/files/res-311-cfe58add7585fbc4.pdf

Consejo Nacional de Educación. (1884). Ley 1.420 de Ley de Educación Común., 8 de julio de 1884. Recuperado de http://www.bnm.me.gov.ar/giga1/documentos/ EL002646.pdf

Frawley, W. (2000). Vygotsky y la Ciencia Cognitiva. Buenos Aires: Paidós.

Frith, U. (2016). Why is it difficult for people with autism to interact with other people? Serious Science. Recuperado de https://youtu.be/N6ylH-LYjOM 
Frith, U., \& Happé, F. (1994). Language and communication in autistic disorders. Philosophical Transactions of the Royal Society of London. Series B: Biological Sciences, 346, 135. https:// doi.org/10.1098/rstb.1994.0133

Grosso, M. L. (2020a). Didáctica inclusiva de la lengua española en la Escuela Secundaria apara Estudiantes con TEA. Hispanista, XXI , 80. Recuperado de http://www.hispanista.com.br/ Rostos/rosto80esp.htm

Grosso, M. L. (2020 $)$. La enseñanza del español como lengua extranjera a Estudiantes con Trastorno del Espectro Autista. Quintú Quimün, 4. Recuperado de http://revele.uncoma.edu. ar/htdoc/revele/index.php/lingustica/issue/view/221

Grosso, M. L. $\left(2020^{\circ}\right)$. La Inclusión en el enfoque glotodidáctico del español del Marco Común Europeo de Referencia para las Lenguas. Hispanista, XXI , 83. Recuperado de http://www. hispanista.com.br/Rostos/rosto80esp.htm

Grosso, M. L. $\left(2021^{\mathrm{d}}\right)$. El Autismo en los manuales diagnósticos internacionales: cambios y consecuencias en las últimas ediciones. Revista española de discapacidad, 9(1), 273-283. Recuperado de https://www.cedd.net/redis/index.php/redis/article/view/743

Hobson, P. (1990). On acquiring knowledge about people and the capacity to pretend: Response to Leslie (1987). Psychological Review, 97(1), 114-121. https://doi.org/10.1037/0033295x.97.1.114

Hobson, P. (1991). Against the theory of 'theory of mind'. British Journal of Developmental Psychology, 9(1), 33-51. https://doi.org/10.1111/j.2044-835X.1991.tb00860.x

Hobson, P. (1993). Autism and the development of mind. UK: Erlbaum Associates Ld Hove.

Honorable Congreso de la Nación Argentina. (1988). Ley 23.592 de Derechos y Garantías Constitucionales. Boletín Nacional del 05 de Septiembre de 1988. Recuperado de https:// www.argentina.gob.ar/normativa/nacional/ley-23592-20465

Honorable Congreso de la Nación Argentina. (1997). Ley 24.901 de Sistema de Prestaciones Básicas en Habilitación y Rehabilitación Integral a Favor de las Personas con Discapacidad. Boletín Nacional de 05 de Diciembre de 1997. Recuperado de https://www.argentina.gob.ar/ normativa/nacional/ley-24901-47677

Honorable Congreso de la Nación Argentina. (2000). Ley 25.280 Apruébase la Convención Interamericana para la Eliminación de Todas las Formas de Discriminación contra las Personas con Discapacidad, suscrita en Guatemala. Boletín Nacional del 04 de Agosto de 2000. Recuperado de https://www.argentina.gob.ar/normativa/nacional/ley-25280-63893

Honorable Congreso de la Nación Argentina. (2001). Ley 25.504 del Certificado de Único de Discapacidad. Boletín Nacional del 13-Dic-2001. Recuperado de https://www.argentina.gob. ar/normativa/nacional/ley-24308-70726

Honorable Congreso de la Nación Argentina. (2006). Ley 26.206 de Educación Nacional. Recuperado de https://www.argentina.gob.ar/educacion/validez-titulos/glosario/ley26206

Honorable Congreso de la Nación Argentina. (2008). Ley 26.378 de Adhesión a la Convención Internacional sobre los Derechos de las Personas con Discapacidad (2007) y su Protocolo Facultativo. Recuperado de http://www.mp.gba.gov.ar/turismo/downloads/Ley Nacional 26.378.pdf 
Honorable Congreso de la Nación Argentina. (2014). Ley 27.043 Declárase de Interés Nacional el abordaje integral e interdisciplinario de las personas que presentan Trastornos del Espectro Autista (TEA). Recuperado de http://www.senado.gov.ar/parlamentario/ parlamentaria/24008/downloadOrdenDia

Honorable Congreso de la Nación Argentina. (2014). Ley 27.044 que Asigna a las Personas con Discapacidad y su Protocolo Facultativo jerarquía constitucional. Boletín Nacional del 22 de Diciembre de 2014. Recuperado de https://webcache.googleusercontent.com/sear ch?q=cache:50Z10rR5d2YJ:https://www.argentina.gob.ar/normativa/nacional/ley-27044$\underline{239860+\& c d=8 \& h l=e s \& c t=c \operatorname{lnk} \& g l=i t \& c l i e n t=\text { safari }}$

Memorándum No 02. (2017). Rectificación Memorándum 01 Asunto: Protocolo de Solicitud de Intervención ante la Detección de Estudiantes con NEDD. Dirección General de Educación Secundaria, Ministerio de Educación de la Provincia de Córdoba. Recuperado de https:// www.cba.gov.ar/memorandum-y-resoluciones-2017/

Kanner, L. (1943). Autistic disturbances of affective contact. Nervous Child, 2, 217-250. Recuperado de http://mail.neurodiversity.com/library kanner 1943.pdf

Ministerio de Educación de la Provincia de Córdoba. (2000). Resolución Ministerial 1114 Certificación final de estudios para Estudiantes con Necesidades Educativas Especiales. Ministerio de Educación de la Provincia de Córdoba, 2000. Recuperado de https://www. igualdadycalidadcba.gov.ar/SIPEC-CBA/PolSocioeducativas/Documentos/Integracion/ ResolucionMinisterialNro-1114.pdf

Ministerio de Educación de la Provincia de Córdoba. (2008). Resolución Ministerial 635 Integración escolar de Estudiantes con NEE. Ministerio de Educación de la Provincia de Córdoba, 19 de diciembre de 2008. Recuperado de https://www.igualdadycalidadcba.gov.ar/SIPEC-CBA/ PolSocioeducativas/Documentos/Integracion/ResolucionMinisterialNro-635.pdf

Ministerio de Educación de la Provincia de Córdoba. (2011). Resolución Ministerial 667 Proceso de integración escolar. Ministerio de Educación de la Provincia de Córdoba, 2011. Recuperado de https://www.igualdadycalidadcba.gov.ar/SIPEC-CBA/PolSocioeducativas/Documentos/ Integracion/Resolucion667.pdf

Ministerio de Educación de la Provincia de Córdoba. (2016). Lineamientos de la política educativa 2016-2019. Provincia de Córdoba, Argentina. Recuperado de https://www. igualdadycalidadcba.gov.ar/SIPEC-CBA/publicaciones/PNFP/MaterialesCba/2016/ Lineamientos-de-la-politica-ed-2016-2019.pdf

Ministerio de Educación de la Provincia de Córdoba. (2016). Resolución Ministerial 311 Ampliación de la Resolución Ministerial 667/11, con el fin de regular los procesos de integración escolar, certificación y titulación de los trayectos de Estudiantes que presentan necesidades educativas derivadas de la discapacidad en el Nivel de Educación Secundaria. Córdoba, 22 de abril de 2016. Recuperado de https://www.igualdadycalidadcba.gov.ar/SIPEC-CBA/ PolSocioeducativas/Documentos/Integracion/2-ResNro311-2016.pdf

Organización de las Naciones Unidas (ONU). (2006). Convención Internacional de los Derechos de las Personas con Discapacidad (CIDPD). En: http://www.un.org/esa/socdev/enable/ documents/tccconvs.pdf

Organización de las Naciones Unidas (ONU). (1989). Convención sobre derechos del niño. Nueva York. Recuperado de https://www.un.org/es/events/childrenday/pdf/derechos.pdf 
Organización de las Naciones Unidas para la Educación y la Cultura (UNESCO).(1990). Declaración Mundial sobre educación para todos y marco de acción para satisfacer las Necesidades Básicas de Aprendizaje. 4-9 de marzo de 1990, Jomtien-Tailandia. Recuperado de https:// unesdoc.unesco.org/ark:/48223/pf0000127583 spa

Organización de las Naciones Unidas para la Educación y la Cultura (UNESCO). (1994). Declaración y Marco de Acción de la Conferencia Mundial sobre Necesidades Educativas Especiales: Acceso y Calidad. 7-10 junio de 1994, Salamanca, España. Recuperado de https://unesdoc. unesco.org/ark:/48223/pf0000110753 spa

Organización Mundial de la Salud. (1990). CIE-10. Décima Edición de la Clasificación Internacional Estadística de Enfermedades y Problemas Relacionados con la Salud. Ginebra: Autor. Recuperado de http://ais.paho.org/classifications/Chapters/

Organizzazione Mondiale della Sanità (OMS). (2001). ICF: International Classification of Functioning, Disability and Health, Genève. OMS; trad. it. Classificazione internazionale del funzionamento, della disabilità e della salute (ICF). Trento: Erickson, 2002.

Organización Mundial de la Salud (OMS). (2018). CIE-11. Undécima Edición de la Clasificación Internacional Estadística de Enfermedades y Problemas Relacionados con la Salud, Ginebra: Autor. Recuperado de https://icd.who.int/browse11/l-m/es

Ozonoff, S., Pennington, B., \& Rogers, S. (1991). Executive function deficits in high-functioning autistic individuals: a relationship to theory of mind. Journal of child psychology and psychiatry, 32(7), 1081-1105. https://doi.org/10.1111/j.1469-7610.1991.tb00351.x

Poder Ejecutivo Nacional. (2019). Decreto del Presidente de la Nación Argentina No 777 Apruébase la Reglamentación de la Ley No 27.043. Boletín Oficial de la República Argentina, 20 de noviembre de 2019. Recuperado de https:// www.boletinoficial.gob.ar/detalleAviso/ primera/221428/20191120

Poder Legislativo Provincial. (2017). Ley No 10.443 de Adhesión de la Provincia de Córdoba a la Ley Nacional No 27.306 de Abordaje Integral e Interdisciplinario de los Sujetos que Presentan DificultadesEspecíficas de Aprendizaje(DEA). Boletín Oficial, 18deOctubrede2017. Recuperado de http://web2.cba.gov.ar/web/leyes.nsf/ 85a69a561f9ea43d03257234006a8594/bb5cbc0 37765565d0325814e00564e53? OpenDocument

Rizzolatti, G., \& Craighero, L. (2004). The mirror neuron system. Annual Review Neuroscience, 27, 169-92. https://doi.org/10.1146/annurev.neuro.27.070203.144230

Vianello, R. (2011). Disabilità cognitive (nell'ottica per cui la comprensione dello sviluppo atipico favorisce anche la comprensione dello sviluppo tipico e più in generale il funzionamento della mente). Padova: UniPD.

Vygotsky, L. (1964). Pensamiento y lenguaje. Buenos Aires: Lautaro.

Wing, L., \& Gould, J. (1979). Severe Impairments of Social Interaction and Associated Abnormalities in Children: Epidemiology and Classification. Journal of Autism and Developmental Disorders, 9, 11-29. https://doi.org/10.1007/bf01531288 